\title{
ERRATUM
}

T. Takamasa $\cdot$ T. Hazuku $\cdot$ N. Fukamachi

N. Tamura $\cdot$ T. Hibiki $\cdot$ M. Ishii

\section{Effect of gravity on axial development of bubbly flow at low liquid Reynolds number}

Published online: 5 April 2005

(C) Springer-Verlag 2005

\section{Exp Fluids (2004) 37:631-644}

$$
\begin{aligned}
\left\langle\left\langle V_{g j}\right\rangle\right\rangle= & \sqrt{2}\left(\frac{M_{F \infty} \sigma}{\rho_{f}^{2}}\right)^{1 / 4} \\
& \times \frac{18.67\left(1-\left\langle\alpha_{g}\right\rangle\right)^{2}\left(\frac{M_{F}}{M_{F \infty}}\right)}{1+17.67\left(1-\left\langle\alpha_{g}\right\rangle\right)^{6 / 7}\left(\frac{M_{F}}{M_{F \infty}}\right)^{3 / 7}}
\end{aligned}
$$

looked in Eq. 10 and Eq. 12
equations are shown below.

$$
\begin{aligned}
\left\langle\left\langle V_{g j}\right\rangle\right\rangle= & \sqrt{2}\left\{\frac{\left(\Delta \rho g_{z}+M_{F \infty}\right) \sigma}{\rho_{f}^{2}}\right\}^{1 / 4} \\
& \times \frac{18.67\left(1-\left\langle\alpha_{g}\right\rangle\right)^{2}\left\{\frac{\Delta \rho g_{z}\left(1-\left\langle\alpha_{g}\right\rangle\right)+M_{F}}{\Delta \rho g_{z}+M_{\mathrm{F} \infty}}\right\}}{1+17.67\left(1-\left\langle\alpha_{g}\right\rangle\right)^{6 / 7}\left\{\frac{\Delta \rho g_{z}\left(1-\left\langle\alpha_{g}\right\rangle\right)+M_{F}}{\Delta \rho g_{z}+M_{F \infty}}\right\}^{3 / 7}}
\end{aligned}
$$

The online version of the original article can be found at http://dx.doi.org/10.1007/s00348-004-0844-9

T. Takamasa $(\bowtie) \cdot$ T. Hazuku $\cdot$ N. Fukamachi $\cdot$ N. Tamura Faculty of Marine Technology,

Tokyo University of Marine Science and Technology,

Etchujima, Koto, 135-8533 Tokyo, Japan

E-mail: takamasa@e.kaiyodai.ac.jp

Tel.: + 81-3-5245-7406

Fax: $+81-3-5245-7410$

T. Hibiki

Research Reactor Institute,

Kyoto University, Kumatori, Sennan,

590-0494 Osaka, Japan

M. Ishii

School of Nuclear Engineering,

Purdue University, 400 Central Drive,

West Lafayette, IN 47907-2017, USA 